\title{
CPTAC Causes of Death Codelist
}

National Cancer Institute

\section{Source}

National Cancer Institute. CPTAC Causes of Death Codelist. NCI Thesaurus. Code C158034.

Terminology used in support of the data collection efforts of the Clinical Proteomic

Tumor Analysis Consortium (CPTAC) with the focus on causes of death. 